\title{
Sicca symptoms and secondary Sjögren's syndrome in systemic lupus erythematosus: comparison with rheumatoid arthritis and correlation with disease variables
}

\author{
I-M Gilboe, T K Kvien, T Uhlig, G Husby
}

\begin{abstract}
Objective-Firstly, to study the prevalence of ocular and oral sicca symptoms, reduced tear and saliva production, and the minimum frequency of secondary Sjögren's syndrome (sSS) in systemic lupus erythematosus (SLE). Secondly, to compare sicca symptoms and findings with those of matched patients with rheumatoid arthritis (RA), and sicca symptoms with those in healthy controls. Finally, to study possible associations of clinical variables with sicca symptoms and sSS in SLE.

Methods-Self reported sicca symptoms were recorded in 81 patients with SLE aged $\leqslant 70,81$ matched patients with $R A$, and 81 matched healthy controls. Other study variables included Schirmer-I test (S1T), unstimulated whole saliva, health status measures (in SLE and RA), disease activity, accumulated organ damage, and serological markers (in SLE).

Results-A significantly higher proportion of patients with SLE reported sicca symptoms than healthy controls. Further, a significantly higher proportion reported ocular sicca symptoms (43 and $21 \%$, respectively) and had pathologically reduced S1T compared with RA (46 and $21 \%$, respectively). No difference was seen in oral sicca symptoms and saliva production. In SLE, sicca symptoms were associated with fatigue, and sSS with anti-SSB or anti-SSA antibodies, or both.

Conclusions-An increased prevalence of sicca symptoms was found in patients with SLE compared with controls, and a higher prevalence of ocular sicca symptoms and reduced tear production in SLE compared with RA. Sicca problems should be considered in the care of patients with SLE, especially those with anti-SSB and/or anti-SSA antibodies who have sicca symptoms and fatigue.
\end{abstract}

(Ann Rheum Dis 2001;60:1103-1109)

Patients with systemic lupus erythematosus (SLE) frequently have sicca symptoms, which may be related to concomitant occurrence of Sjögren's syndrome (SS). The relation between SLE and SS was first reported by Heaton in $1959,{ }^{1}$ who considered SS to be a mild form of SLE. Later, SS was classified as a distinct entity, named primary SS (pSS), or occurring as secondary SS (sSS) in association with other autoimmune diseases. ${ }^{2}$
The prevalence of sSS in SLE is reportedly from 8 to $30 \%$ in different studies. ${ }^{3-5}$ The variation is mainly explained by application of different classification criteria and possibly also by different patient selection. The preliminary European classification criteria for SS require one or more sicca symptoms and at least two objective findings: one pathological finding indicating eye involvement and one for oral involvement. These criteria have been found to possess satisfactory specificity for a definite diagnosis of sSS. ${ }^{6}$ The use of a sicca symptoms questionnaire has been useful for the identification of patients suggested to have $\mathrm{SS}^{7}$ the Schirmer-I test (S1T) for identification of ocular disease, and the unstimulated whole saliva (UWS) flow examination for oral involvement. All these diagnostic items are included in the European classification criteria, ${ }^{8}$ have shown a satisfactory balance between sensitivity and specificity, ${ }^{6}$ and are feasible in daily practice.

Health status, disease activity, and accumulated organ damage are accepted as important independent dimensions of outcome in SLE, ${ }^{,-11}$ and are recommended for use in SLE studies. ${ }^{12}$ Secondary SS may affect health status as well as organ damage. Previously, only one study has compared functional disability and end organ damage between patients with SLE with and without sSS. ${ }^{13}$ Although Sjögren originally considered rheumatoid arthritis (RA) to be the most common of all connective tissue diseases associated with $\mathrm{SS},{ }^{14}$ no study has so far directly compared sicca symptoms and findings in patients with SLE and RA matched for sex, age, and disease duration.

The main objective of this study was, firstly, to study the prevalence of ocular and oral sicca symptoms, reduced saliva and tear production, and estimate a minimum frequency of $\mathrm{sSS}^{6}$ in SLE. Secondly, to compare the findings in patients with SLE with those in patients with RA matched for sex, age, and disease duration; and, thirdly, to compare the occurrence of sicca symptoms with that in matched healthy controls. We also wanted to examine associations between sicca symptoms and other findings compatible with sSS, on the one hand, and clinical and health status variables in SLE, on the other.

Patients and methods

SETTING

The study was carried out at the Oslo City Department of Rheumatology, Diakonhjemmet Hospital, which offers a rheumatological 
service for the community of Oslo, the capital city of Norway with approximately 500000 inhabitants. Previous studies have shown that the community of Oslo is a reliable setting for epidemiological studies in rheumatology. ${ }^{15} 16$

PATIENTS AND HEALTHY CONTROLS

A cohort of 93 patients with SLE fulfilling the revised classification criteria for SLE, ${ }^{17}$ and with a residential address in Oslo, has been followed up longitudinally since $1995-96 .{ }^{18}$ Eighty seven of them participated in a follow up examination in 1997-98, of whom 81 patients aged $\leqslant 70$ were eligible for the present study.

Eighty one patients with a diagnosis of $\mathrm{RA}^{19}$ from the Oslo RA register at the Diakonhjemmet Hospital were matched for sex, age $( \pm 2$ years), and disease duration ( \pm 2 years) with the patients with SLE. The register was established in 1994, and is continuously updated for patients with RA with disease onset after the age of 16 and with a residential address in Oslo. ${ }^{15}$

As healthy controls, subjects were selected from the population register of Oslo. The controls received an invitation by mail, and the initial non-respondents received a reminder after two weeks. We obtained data on sicca symptoms from 81 subjects, each being matched for age, sex, and geographical area in Oslo with one of the patients with SLE. None of the healthy controls had rheumatic disease.

DATA COLLECTION

Data were collected in 1997-98 for the patients with SLE and in 1996-97 for the patients with RA through clinical examination, laboratory analyses, self reported questionnaire, standardised interview, and careful review of the hospital records.

The clinical examination of the patients with SLE and RA consenting to participate included tear (S1T) and saliva (UWS) production and assessment of the 28 tender and 28 swollen joint count. Further, the SLE Disease Activity Index (SLEDAI),${ }^{9}$ accumulated organ damage (the Systemic Lupus International Collaborating Clinics/American College of Rheumatology Damage Index (SLICC/ACRDI) $),{ }^{20}$ and the corresponding laboratory analyses were assessed in patients with SLE. S1T and UWS were performed by two specially trained nurses in the patients with SLE and RA under the continuous supervision of a rheumatologist (IMG or TU). The remaining clinical examination was performed by one of us (IMG) for the patients with SLE, and by a trained research nurse in collaboration with a rheumatologist (TU) for the patients with RA. ${ }^{21}$

The self reported questionnaire was completed the day before or during the visit for the patients with SLE and RA and collected by mail in 1997-98 for the healthy controls. It comprised questions about oral and ocular sicca symptoms, ${ }^{6}$ and self reported health status measures (Modified Health Assessment Questionnaire (MHAQ), ${ }^{22}$ MOS Short Form-36 (SF-36), ${ }^{23} 100 \mathrm{~mm}$ visual analogue scales (VAS) on pain and fatigue, and patient's global assessment of disease activity). The interview included smoking habits, previous and current use of antirheumatic drugs, and current use of drugs able to induce sicca symptoms - that is, xerogenic drugs ( $\beta$ blockers, diuretics, antidepressants, neuroleptics, and antihistamines)

\section{ASSESSMENTS AND INSTRUMENTS}

The S1T was performed according to published guidelines. ${ }^{24}$ Patients had not used tear substitution for at least one hour before the examination. The standardised tear test strips, always starting with the right eye, were placed between the medial and the lateral part of the lower eyelid, without preceding use of anaesthetic eye drops. The patients sat with their eyes closed. After five minutes the strips were removed and the length of the wetted area of the strip was measured, starting from the notch corresponding to the inferior lid margin. Tear production was pathological if the length of the wetted area was $\leqslant 5 \mathrm{~mm}$ at one or both eyes. ${ }^{8}$

The UWS test was performed in the morning. ${ }^{24}$ Patients had not eaten, smoked, swallowed liquids, or rinsed their mouths for at least one hour before the test. The patients were seated, inclining slightly forward and protected from gustatory or other stimulation during the test. After swallowing, the saliva was collected over 15 minutes by passive spitting into a preweighed container. Flow rate was expressed as $\mathrm{ml} / 15 \mathrm{~min}(1 \mathrm{~g}=1 \mathrm{ml})$. Reduced saliva production was recorded if the saliva production was $1.5 \mathrm{ml} / 15 \mathrm{~min}$ or less. ${ }^{8}$

Questions on oral and ocular dryness were identical with those from the European classification criteria for SS (three questions each on eyes and mouth), ${ }^{6}$ accepted as the standard questionnaire to be used for the preliminary selection of potential patients with sicca symptoms in epidemiological surveys. Keratoconjuntivitis sicca was defined as one or more ocular sicca symptoms in combination with pathological S1T, and xerostomia as one or more oral sicca symptoms in combination with pathological UWS.

The SLEDAI is a validated disease activity measure index. ${ }^{9}$ The version covering the past 10 days was used. It contains 24 descriptors in nine organ systems, including clinical and laboratory measures of SLE activity, and is weighted to reflect the degree of activity. The maximum possible score is 105 .

The SLICC/ACR-DI is designed to assess accumulated organ damage in patients with SLE since onset of the disease, caused by the disease itself or the treatment. ${ }^{20}$ Damage is defined for 12 organ systems, and the score can only increase over time, theoretically to a maximum of 47.

The MHAQ is a modified shortened version of the Stanford Health Assessment Questionnaire, ${ }^{22}$ examining eight dimensions of difficulties with the performance of activities of daily living, on a scale of $1-4$. The SF-36 is a generic instrument, measuring eight dimensions of health status. ${ }^{23}$ The scales of the SF-36 have 
values from 0 to 100, with a higher value representing better functioning/health. The version covering the past four weeks was used.

Antibodies were examined routinely by the laboratory; anti ds-DNA by Crithidia luciliae indirect immunofluorescence and anti-SSA and anti-SSB antibodies by an enzyme linked immunosorbent assay (ELISA) test.

Results of a rheumatoid factor (RF) test in patients with RA were obtained from the hospital records. RF positivity was defined as a titre of the Waaler-Rose $\operatorname{IgM} \mathrm{RF} \geqslant 64$ on at least one occasion.

Disease onset was defined as the time when the patients fulfilled the ARA/ACR criteria for the respective disease (SLE or RA), and disease duration as the period from the disease onset to the time of this study.

\section{DATA ANALYSES AND STATISTICS}

Tear and saliva production, as well as the proportions of patients with pathological tear and saliva production, keratoconjunctivitis sicca, xerostomia, and sSS, was compared for matched patients with SLE and RA; the proportion with sicca symptoms for patients with SLE was compared with matched patients with RA and healthy controls.

All statistical analyses were performed by SPSS, version 8.0. Descriptive statistics are presented as means with range or standard deviation (SD) for continuous variables, as counts and percentage for categorical variables. In matched patients and controls paired analyses were used when comparing continuous variables and the McNemar test when comparing categorical variables. Student's $t$ tests for independent samples were used to compare the means of two SLE groups, and the $\chi^{2}$ test for counts. The differences were regarded as significant for $\mathrm{p}<0.05$ and highly significant for $\mathrm{p}<0.01$. Correlation was examined by the Pearson correlation coefficient. A correlation was considered as strong if the correlation coefficient was $>0.70$, moderate to substantial if $0.30-0.70$, and weak if $<0.30$. Demographic and disease variables were used as independent variables when examining association with sicca symptoms (no sicca symptom $v$ one or more sicca symptoms) and sSS in SLE (no sSS $v \mathrm{sSS}$ ). Variables to be used in the multivariate logistic regression analyses were identified in bivariate analyses if $\mathrm{p}<0.15$. If candidate variables were strongly related-for example, VAS fatigue and SF-36 vitality, one variable only was selected.

ETHICS AND LEGAL ASPECTS

The local ethical committee approved the study and the data inspectorate the register of patients with SLE and RA in Oslo.

\section{Results}

PATIENTS

Table 1 gives demographic and disease variables for 81 patients with SLE, 81 matched patients with RA, and 81 matched healthy controls. Eighty nine per cent were women in all three groups, and other demographic characteristics were also comparable. Significantly fewer patients with SLE used non-steroidal anti-inflammatory drugs (NSAIDs) than did patients with RA. Differences in the use of xerogenic drugs were not statistically significant. The mean number of tender and swollen joints was significantly higher in RA than in SLE, and the patients with RA had worse physical disability scores (MHAQ and SF-36 physical) than those with SLE, whereas other disease and health status measures were similar in the two groups (table 1).

Table 1 Demographic variables, disease, and health status measures of the study groups. (Mean (range) for continuous, number (\%) for categorical variables)

\begin{tabular}{|c|c|c|c|c|c|c|}
\hline & \multicolumn{2}{|c|}{$S L E(n=81)$} & \multicolumn{2}{|c|}{$R A(n=81)$} & \multicolumn{2}{|c|}{ Controls $(n=81)$} \\
\hline \multicolumn{7}{|l|}{ Demographics } \\
\hline Women & 72 & $(89)$ & 72 & $(89)$ & 72 & (89) \\
\hline Age (years) & 44 & $(20-70)$ & 44 & $(22-69)$ & 44 & $(20-70)$ \\
\hline White subjects & 81 & $(100)$ & 81 & $(100)$ & 81 & $(100)$ \\
\hline \multicolumn{7}{|l|}{ Disease variables } \\
\hline Disease duration (years) & 7.8 & $(2-27)$ & 7.8 & $(2-26)$ & NA & \\
\hline Smokers (current) & 27 & (33) & 30 & $(37)$ & 21 & (26) \\
\hline Xerogenic drugs & 17 & (21) & 10 & (12) & NA & \\
\hline \multicolumn{7}{|c|}{ Current antirheumatic drugs } \\
\hline NSAIDs $\ddagger$ & & $(21) \dagger$ & 38 & $(47)$ & NA & \\
\hline Glucocorticosteroids & 48 & (59) & 36 & $(44)$ & NA & \\
\hline DMARDs $\ddagger$ & 30 & (37) & 24 & (30) & NA & \\
\hline Cytotoxic drugs & 15 & (19) & 22 & (27) & NA & \\
\hline SLEDAI $\ddagger$ & 6.67 & $7(0-24)$ & NA & & NA & \\
\hline SDI $\ddagger$ & 1.91 & $1(0-12)$ & NA & & NA & \\
\hline RF positivity & NA & & & $(43)$ & NA & \\
\hline 28 Tender joint count & 3.5 & $(0-28) \dagger$ & 6.4 & $(0-23)$ & NA & \\
\hline 28 Swollen joint count & 2.0 & $(0-21) \dagger$ & 7.5 & $(0-28)$ & NA & \\
\hline \multicolumn{7}{|l|}{ Health status measures } \\
\hline MHAQ $\ddagger$ & 1.32 & $2(1.0-3.00) \dagger$ & 1.52 & $2(1.0-2.75)$ & 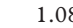 & $8(1.0-2.50) \dagger$ \\
\hline SF-36 physical & 67.2 & $(5-100)^{\star}$ & 59.1 & $(0-100)$ & 88.9 & $(10-100) \dagger$ \\
\hline SF-36 pain & 51.8 & $(0-100)$ & 49.0 & $(0-100)$ & 76.4 & $(0-100) \dagger$ \\
\hline SF-36 general health & 45.5 & $(5-100)$ & 47.9 & $(0-100)$ & 77.0 & $(20-100) \dagger$ \\
\hline SF-36 vitality & 38.5 & $(0-100)$ & 44.0 & $(5-80)$ & 59.4 & $(0-100) \dagger$ \\
\hline SF-36 mental & 71.1 & $(16-100)$ & 73.9 & $(12-100)$ & 77.3 & $(0-100)^{\star}$ \\
\hline
\end{tabular}


Table 2 Sicca symptoms (proportions, \%) in 81 matched patients with SLE, RA and healthy controls

\begin{tabular}{llll}
\hline & $\begin{array}{l}S L E \\
(n=81)\end{array}$ & $\begin{array}{l}R A \\
(n=81)\end{array}$ & $\begin{array}{c}\text { Controls } \\
(n=81)\end{array}$ \\
\hline $\begin{array}{l}\text { Dry eyes daily for more than 3 months? } \\
\text { Sensation of sand or gravel? }\end{array}$ & $28^{\star}$ & 14 & $10 \dagger$ \\
Use of tear substitutes more than 3 times a day? & $41^{\star}$ & 24 & $13 \dagger$ \\
Dry mouth daily for more than 3 months? & 37 & 1 & 2 \\
Experienced swollen salivary glands? & 11 & 30 & $9 \dagger$ \\
Drink liquids to swallow dry food? & 47 & 6 & $1 \dagger$ \\
Combinations: & 61 & 52 & $19 \dagger$ \\
At least one sicca symptom & $43^{\star}$ & 27 & $24 \dagger$ \\
At least one sicca eye symptom & 52 & 42 & $16 \dagger$ \\
At least one sicca mouth symptom & $34^{\star}$ & 17 & $9 \dagger$ \\
At least one sicca symptom from eye and mouth & $34^{\star}$ & & 9 \\
\hline
\end{tabular}

${ }^{\star} \mathrm{p}<0.05$ SLE $v$ RA (McNemar test for paired samples).

$\mathrm{t} \mathrm{p}<0.01$ SLE $v$ healthy controls (McNemar test for paired samples).

Table 3 Tear and saliva production (mean (SD)) in matched patients with SLE and RA

\begin{tabular}{lccc}
\hline & $S L E(n=81)$ & $R A(n=81)$ & $p$ Value $^{*}$ \\
\hline Schirmer-I test right eye $(\mathrm{mm} / 15 \mathrm{~min})$ & $14.4(13.1)$ & $18.9(13.3)$ & 0.04 \\
Schirmer-I test left eye $(\mathrm{mm} / 15 \mathrm{~min})$ & $15.4(13.1)$ & $22.9(12.4)$ & $<0.001$ \\
Unstimulated whole saliva $(\mathrm{ml} / 15 \mathrm{~min})$ & $4.30(3.54)$ & $4.46(3.12)$ & 0.76 \\
\hline
\end{tabular}

^Paired analyses.

SICCA SYMPTOMS IN SLE, RA, AND MATCHED HEALTHY CONTROLS

Table 2 presents the proportions of patients with SLE, patients with RA, and healthy controls who gave positive answers to the six sicca symptom questions included in the European classification criteria for SS. A significantly higher proportion of the patients with SLE compared with healthy controls gave positive answers to each of the three different ocular and oral sicca questions except for the question "use of tear substitutes more than three times a day". Further, the four examined combinations of sicca symptoms were seen more commonly in SLE (table 2).

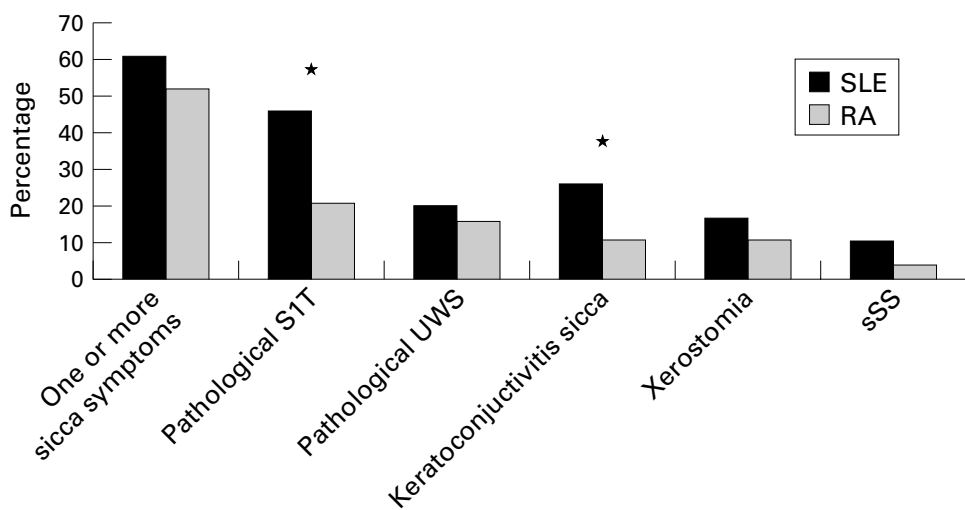

Figure 1 Proportions of matched patients with SLE and RA with one or more sicca symptoms, pathological reduced Schirmer-I test (S1T), and unstimulated whole saliva (UWS), keratoconjunctivitis sicca, xerostomia, and secondary Sjögren's syndrome (sSS). Keratoconjuntivitis sicca $=$ one or more eye sicca symptoms in combination with pathological S1T; xerostomia = one or more mouth sicca symptoms in combination with pathological UWS. ${ }^{*} p<0.05 S L E v R A$ (McNemar test).

Table 4 Correlations between sicca symptoms, unstimulated whole saliva (UWS), and Schirmers-I test (S1T) in patients with SLE (Pearson coefficient)

\begin{tabular}{lcccccc}
\hline & Age & Sex & $\begin{array}{l}\text { No of sicca } \\
\text { symptoms }\end{array}$ & UWS & S1T right & S1T left \\
\hline Age & 1.00 & & & & & \\
Sex & 0.02 & 1.00 & & & & \\
No of sicca symptoms & 0.07 & 0.12 & 1.00 & & & \\
UWS & -0.23 & -0.27 & -0.31 & 1.00 & & \\
S1T right & -0.10 & -0.05 & -0.12 & 0.13 & 1.00 & \multirow{2}{*}{0.00} \\
S1T left & -0.16 & 0.04 & -0.19 & 0.05 & 0.78 & \\
\hline
\end{tabular}

The prevalence of ocular sicca symptoms was significantly higher in SLE than in RA, as was the combined report of sicca symptoms from eyes and mouth (table 2). The distribution of the three oral sicca symptoms was similar in the two patient groups.

SALIVA AND TEAR PRODUCTION IN SLE COMPARED WITH RA

The mean tear production as measured by the S1T test was significantly lower in both eyes in SLE compared with patients with RA (table 3). A higher proportion of the patients with SLE $(46 \%)$ had reduced tear production compared with RA (21\%) (fig 1). The UWS flow rate (table 3) and proportion of patients with reduced UWS were similar in SLE and RA (20 and $16 \%$, respectively (fig 1)). Keratoconjunctivitis sicca was observed significantly more often in SLE than in RA, 26 and 11\% respectively, whereas the occurrence of xerostomia was similar in the two patient groups, 17 and $11 \%$, respectively (fig 1 ).

Nine patients with SLE (11\%) and three patients with RA (4\%) had one or more sicca symptoms as well as pathologically reduced tear and saliva production, thus fulfilling the European criteria for sSS (fig 1). This result indicates a greater trend towards sSS in SLE than in RA ( $p=0.07)$.

CORRELATION BETWEEN SICCA SYMPTOMS AND FINDINGS IN SLE

Table 4 shows the correlation between sicca symptoms and findings in SLE. Saliva flow correlated weakly with age, sex, and number of sicca symptoms $(r=0.23-0.31)$, whereas the S1T test did not. Oral sicca symptoms ("dry mouth daily for more than three months" and "drink liquid to swallow dry food") correlated better with saliva production than they did with tear production (data not shown).

COMPARISON OF DEMOGRAPHIC AND DISEASE VARIABLES IN PATIENTS WITH SLE WITH AND WITHOUT SICCA SYMPTOMS OR SSS

Table 5 compares the patients with SLE with and without one or more sicca symptoms, and compares the patients fulfilling the classification of sSS and those who did not.

The group with one or more sicca symptoms $(\mathrm{n}=49,61 \%)$ used xerogenic drugs more frequently, had a higher level of organ damage score, significantly lower scores of SF-36 subscales (except SF-36 mental), and higher levels of VAS pain and fatigue than the group of patients without sicca symptoms. Otherwise, demographic and disease variables were similar. In logistic regression analyses one or more sicca symptoms was independently associated with VAS fatigue/SF-36 vitality, whereas organ damage index (SLICC/ACR-DI) and current use of xerogenic drugs had a trend of association (table 6). None of the other variables showing bivariate associations remained in the final multivariate model.

None of the nine patients with SLE fulfilling the European classification criteria for sSS (called the sSS group) had renal disorder. Further, in the sSS group a higher proportion was 
Table 5 Comparisons of demographic, disease variables, and health status measures between patients with SLE with and without at least one sicca symptom and sSS. Mean (SD) for continuous, number (\%) for categorical variables

\begin{tabular}{|c|c|c|c|c|c|c|c|c|}
\hline & \multicolumn{4}{|c|}{ Sicca symptoms } & \multicolumn{4}{|c|}{ Secondary Sjögren's syndrome } \\
\hline & \multicolumn{2}{|c|}{ Present $(n=49)$} & \multicolumn{2}{|c|}{ Absent $(n=32)$} & \multicolumn{2}{|c|}{ Present $(n=9)$} & \multicolumn{2}{|c|}{ Absent $(n=72)$} \\
\hline \multicolumn{9}{|l|}{ Demographics } \\
\hline Female sex & 44 & $(90)$ & 28 & $(88)$ & 9 & $(100)$ & 63 & $(88)$ \\
\hline Age & 44.8 & $(12.7)$ & 44.1 & $(12.4)$ & 48.6 & $(11.1)$ & 44.0 & $(12.6)$ \\
\hline Smokers (current) & 17 & (35) & 9 & (29) & 4 & $(44)$ & 22 & (31) \\
\hline Xerogenic drugs & 14 & $(29)^{\star}$ & 3 & (9) & 3 & (33) & 14 & (19) \\
\hline \multicolumn{9}{|l|}{ Disease variables } \\
\hline Age at disease onset & 34.6 & $(13.4)$ & 36.8 & $(12.7)$ & 37.5 & $(11.8)$ & 35.2 & $(13.3)$ \\
\hline Disease duration & 8.3 & $(5.5)$ & 7.0 & $(4.7)$ & 8.0 & $(5.1)$ & 7.2 & $(5.3)$ \\
\hline Disease activity (SLEDAI) & 6.67 & $7(5.5)$ & 6.68 & $8(5.9)$ & 6.67 & $7(6.0)$ & 6.68 & $8(5.6)$ \\
\hline Organ damage (SLICC/ACR-DI) & 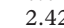 & $2(2.4)^{\star}$ & 1.13 & $3(1.5)$ & 1.89 & $9(3.8)$ & 1.91 & $1(1.9)$ \\
\hline Number of ARA/ACR criteria & 5.49 & $9(1.4)$ & 5.53 & $3(1.2)$ & 5.4 & $(0.9)$ & 5.5 & (1.4) \\
\hline $\operatorname{ESR}(\mathrm{mm} / 1 \mathrm{st} \mathrm{h})$ & 26.5 & $(20.3)$ & 22.1 & $(20.9)$ & 26.1 & $(11.2)$ & 24.6 & $(20.4)$ \\
\hline Anti-dsDNA antibody & 29 & $(59)$ & 18 & $(56)$ & 4 & $(44)$ & 43 & $(60)$ \\
\hline Anti-SSA antibody & 16 & (33) & 16 & $(50)$ & 8 & $(89)^{\star}$ & 26 & (36) \\
\hline Anti-SSB antibody & 8 & $(16)$ & 5 & (16) & 5 & $(56)^{\star}$ & 8 & (11) \\
\hline C3 decreased $(<0.67 \mathrm{~g} / 1)$ & 4 & $(8)$ & 4 & (13) & 1 & (11) & 7 & $(10)$ \\
\hline $\mathrm{C} 4$ decreased $(<0.13 \mathrm{~g} / \mathrm{l})$ & 18 & (37) & 11 & (34) & 5 & $(56)$ & 24 & (33) \\
\hline Renal disorder & 8 & $(16)$ & 6 & (19) & $0^{\star}$ & & 14 & (19) \\
\hline \multicolumn{9}{|l|}{ Health status measures } \\
\hline SF-36 physical & 61.6 & $(27.1)^{\star}$ & 75.8 & $(21.9)$ & 58.9 & $(33.7)$ & 68.2 & $(24.9)$ \\
\hline SF-36 bodily pain & 47.1 & $(23.8)^{\star}$ & 58.9 & $(27.6)$ & 51.3 & $(31.7)$ & 51.8 & $(25.3)$ \\
\hline SF-36 vitality & 33.2 & $(22.2)^{\star}$ & 46.5 & $(21.0)$ & 25.6 & $(18.8)^{\star}$ & 40.1 & $(22.5)$ \\
\hline SF-36 general health & 40.3 & $(25.5)^{\star}$ & 53.5 & $(27.8)$ & 39.9 & $(25.3)$ & 46.2 & $(27.3)$ \\
\hline SF-36 mental & 69.8 & $(17.9)$ & 73.0 & $(16.0)$ & 76.0 & (12.9) & 70.5 & $(17.6)$ \\
\hline SF-36 social & 64.5 & $(23.4)^{\star}$ & 75.4 & $(24.1)$ & 61.1 & $(20.2)$ & 69.8 & $(24.5)$ \\
\hline MHAQ & 1.44 & $4(0.49)^{\star}$ & 1.12 & $2(0.24)$ & 1.44 & $4(0.53)$ & 1.30 & $0(0.43)$ \\
\hline VAS pain & 37.7 & $(21.3)^{\star}$ & 26.3 & $(23.9)$ & 31.9 & $(25.6)$ & 33.4 & $(22.8)$ \\
\hline VAS fatigue & 60.6 & $(26.3)^{\star}$ & 38.9 & $(30.3)$ & 68.7 & $(23.1)^{\star}$ & 48.6 & (29.9) \\
\hline Patient global assessment & 2.73 & $3(0.91)$ & 2.44 & $4(1.01)$ & 2.78 & $8(0.83)$ & 2.60 & $0(0.97)$ \\
\hline
\end{tabular}

Two sample $t$ test for continuous and $\chi^{2}$ for categorical variables.

${ }^{\star} \mathrm{p}<0.05$.

Table 6 Associations between the presence of sicca symptoms and secondary Sjögren's syndrome (dependent variables) and disease variables (independent variables) in SLE using multiple logistic regression analyses (odds ratios (OR) with $95 \%$ confidence intervals (CI) and $p$ values)

\begin{tabular}{llll}
\hline & OR & CI & $p$ Value \\
\hline One or more sicca symptoms & & & \\
$\quad$ Use of xerogenic drugs & 3.58 & 0.82 to 15.59 & 0.09 \\
SLICC/ACR-DI & 1.41 & 0.96 to 2.06 & 0.08 \\
SF-36 physical & 1.00 & 0.97 to 1.02 & 0.65 \\
SF-36 pain & 0.99 & 0.97 to 1.03 & 0.91 \\
Fatigue VAS & 1.03 & 1.01 to 1.05 & 0.01 \\
Secondary Sjögren's syndrome & 15.49 & 2.78 to 86.45 & 0.002 \\
$\quad$ Anti-SSB antibody & 1.03 & 0.99 to 1.06 & 0.09 \\
VAS fatigue & 1.03 & \\
\hline
\end{tabular}

positive for anti-SSA and anti-SSB antibodies, they had a lower SF-36 vitality score, and a higher VAS fatigue score. Logistic regression analyses were performed to identify the most important variables associated with sSS in SLE. As all the patients with anti-SSB also had anti-SSA, anti-SSA was not entered into the final model. In the multivariate analysis sSS was significantly associated with anti-SSB antibodies, whereas VAS fatigue had a trend of association (table 6).

\section{Discussion}

The main message of our study is that the patients with SLE have longer lasting ocular and oral sicca symptoms than matched healthy controls, and more ocular sicca symptoms and reduced tear production than matched patients with RA. The clinical consequences are reduced oral and ocular health in SLE ${ }^{25-27}$ and, probably also, sicca symptoms from other exocrine glands, ${ }^{28}$ as oral and ocular autoimmune exocrinopathy has been found to correlate with pathology in exocrine glands elsewhere. ${ }^{28}$ Although a relatively small number of the patients with SLE and RA met the classification criteria for sSS, a high proportion had sicca symptoms and signs of an exocrinopathy, which should be a concern in the management of both patients with SLE and RA.

The proportion of patients with SLE with sicca symptoms in our study compared well with results from some previous studies ${ }^{30-32}$ and was somewhat higher than one study. ${ }^{4}$ Ocular involvement was more common than others have observed, ${ }^{42} 33$ whereas oral involvement was less common than found by Alarcon-Segovia et $a b^{30}$ and Moutsopoulos et $a l .{ }^{32}$ Such differences may be partly explained by different study groups and different assessments.

Our study indicates that sSS is at least as common in SLE as in RA, and Sjögren's suggestion that RA is the chief underlying disorder for $\mathrm{sSS}^{14}$ may be disputed. However, at that time SLE was probably less well recognised as a disease entity than RA. Furthermore, the more frequent ocular sicca symptoms and findings in SLE, indicating more severely affected lachrymal glands, seem to support Vitali's suggestion ${ }^{8}$ that SS associated with SLE is more similar to pSS than to sSS in patients with RA. As our study was designed with age matched groups, this excludes demographic variables as an explanation for this difference in ocular dryness. Age is otherwise known to influence both tear and saliva production and composition. ${ }^{34-36}$ However, this is to our knowledge the first study directly comparing sicca symptoms and findings in matched patients with SLE and RA, and the findings need to be confirmed by others. Concomitant factors may contribute to dryness in SLE and RA, especially depression, ${ }^{37}$ diabetes mellitus, ${ }^{38}$ and 
use of xerogenic drugs. ${ }^{29}$ In this study the proportions of patients with depression were numerically higher in SLE than in RA $(8 \% v$ $3 \%$, respectively), whereas the proportions of patients with diabetes mellitus and use of xerogenic drugs were similar.

Relatively few patients with SLE and RA had swollen salivary glands and/or used tear substitutes, and the two questions out of the six used to examine for sicca symptoms (table 2) did not contribute much to the diagnosis of sSS in our study. Furthermore, the low use of tear substitutes indicates rather mild ocular sicca symptoms in both diseases, which is in line with previous sSS studies ${ }^{4313940}$ and in contrast with the situation in pSS. ${ }^{41}$

Although sicca symptoms and finding were common in both SLE and RA in this study, only a few patients met the European classification criteria for sSS. The prevalence of sSS presented is a minimum estimate, as an incomplete number of relevant tests and examinations, especially minor salivary gland biopsy, were recorded. Characteristic pathological biopsy findings are often reported in SLE, ranging from 20 to $90 \%$ in different studies. ${ }^{4} 3032$

The finding that $32 \%$ of the patients with SLE had subjective oral sicca symptoms without corresponding reduction of UWS suggests that not only a decreased quantity of saliva but also a change in its quality or other local factors may induce a feeling of dryness, known from other studies as well. ${ }^{35} 42$ This low correlation between sicca symptoms and findings has also been observed by others. ${ }^{43}$ Our study supports findings in some, ${ }^{4244}$ but not all previous studies $^{5}$ of a correlation between sSS and antibodies to SSA and SSB in SLE, also seen in patients with progressive systemic sclerosis with sSS ${ }^{45}$ and in otherwise normal adults with dry eyes and mouth. ${ }^{46}$ That fatigue/reduced vitality was the only parameter of health status associated with sicca symptoms and which had a trend towards an association with sSS, supports the suggestion that fatigue is an important feature of both $\mathrm{SS}^{13}{ }^{41}$ and SLE. ${ }^{47}$ Similar findings have been shown in $\mathrm{RA}^{21}$ whereas Sutcliffe et al showed similar fatigue levels in SLE with and without sSS. ${ }^{13}$

Although the patients with SLE with sSS had less renal disease according to the ACR criteria than those in the non-sSS group, neither disease activity nor organ damage differed between the two groups with the test instruments used. ${ }^{45}{ }^{13}$ For example, an association between neuropsychiatric symptoms and sSS was not found in our study, which contrasted with the study of Utset Olsen et al. ${ }^{48}$ However, affected exocrine glands in SLE may contribute to damage not included in the organ damage index. ${ }^{20}$ Oral manifestations like recurrent infections or mouth ulcers, severe gum disease, or excessive dental caries have been found frequently in SLE ${ }^{49-51}$ as well as in pSS. ${ }^{13}$

SLE with sSS may be difficult to distinguish from pSS both clinically and serologically, especially in patients with SLE with onset at an older age. ${ }^{52}$ Although the patients have been diagnosed with SLE, the high proportion of patients with anti-SSA/B antibodies may indicate that some of the anti-dsDNA antibody negative patients might have been given alternative diagnoses.

The methods (S1T and UWS) chosen to assess exocrine gland dysfunction instead of alternative tests-for example, the van Bijsterveld score, tear break up time, or stimulated saliva flow, may be disputed. However, all tests have their advantages and weaknesses. For example S1T is a weak diagnostic test, ${ }^{53-55}$ but was preferred in some epidemiological studies. ${ }^{4356}$ Unstimulated rather than stimulated saliva was preferred in the criteria for SS. ${ }^{6}$

A strength of our study is the successful demographic matches of the study groups, permitting a case-control design. When matching patients with SLE and RA, we controlled for two important demographic variablesnamely, age and disease duration. The data of patients with RA from the register are suggested to be representative for the underlying RA population in Oslo. Our healthy controls reported a somewhat similar prevalence of ocular and oral sicca symptoms compared with a population based study from the United Kingdom, ${ }^{43}$ and higher than that of women without RA in a Greek study. ${ }^{57}$

Limitations of our study are the relatively small samples of patients and controls and the lack of data on tear and saliva production of the latter group. We have previously compared saliva production between 20 healthy controls and 20 of our patients with SLE ${ }^{25}$ and found reduced salivary flow rate in SLE, which is also in accord with other studies. ${ }^{58}{ }^{59}$ Furthermore, a clear weakness of this study is the lack of histological confirmation of SS in the patients with sSS.

The practical clinical conclusions of our study are the significance of sicca symptoms and signs of an exocrinopathy in both SLE and RA, their consequence for treatment and follow up, and the high risk of sSS in patients with SLE with anti-SSB and/or anti-SSA antibodies, particularly in those with sicca symptoms and fatigue.

The authors acknowledge the work of the nurses Ingerid Müller and Sidsel Arnkværn for their assistance with data collection, Valborg Frøysnes for data entry, and Petter Mowinkel for his help in the statistical analyses.

The study is in part supported by grants from the Lions Clubs International MD 104 Norway, the Norwegian Rheumatism Association, the Norwegian Research Council, the Norwegian Women's Public Health Association, the Legacy of Grethe Harbitz, and the Legacy of Trygve Gythfeldt and Wife.

1 Heaton J. Sjögren's syndrome and systemic lupus erythematosus. BMJ 1959;i:466-9.

2 Moutsopoulos H, Webber B, Vlagopoulos T, Chused T, Decker J. Differences in the clinical manifestations of sicca syndrome in the presence and absence of rheumatoid arthritis. Am J Med 1979;66:733-6.

3 Grennan D, Ferguson M, Williamson J, Mavrikakis M, Dick W, Buchanan W. Sjögren's syndrome in SLE: Part 1. The frequency of the clinical and subclinical features of Sjögren's syndrome in patients with SLE. N Z Med J 1977; 86:374-6.

4 Andonopoulos A, Skopouli F, Dimou G, Drosus A, Moutsopoulos H. Sjögren's syndrome in systemic lupus erythematosus. J Rheumatol 1990;17:201-4.

5 Nossent JC, Swaak A. Systemic lupus erythematosus. VII: Frequency and impact of secondary Sjögren's syndrome. Lupus 1998;7:231-4.

6 Vitali C, Bombardieri S, Moutsopoulos H, Balestrieri G, Bencivelli W, Bernstein RM, et al. Preliminary criteria for Benciveli W, Bernstein RM, et al. Preliminary criteria for
the classification of Sjögren's syndrome. Results of a prospective concerted action supported by the European community. Arthritis Rheum 1993;36:340-7. 
7 Brun JG, Jacobsen H, Kloster R, Cuida M, Johannesen AC, Høyeraal HM. Use of sicca symptoms questionnaire for the identification of patients with Sjögren's syndrome in a heterogeneous hospital population with various

8 Vitali C, Moutsopoulos HM, Bombardieri S, and the European Community Study Group on the Diagnostic Criteria for Sjögren's syndrome. The European Community Study Group on the Diagnostic Criteria for Sjögren's syndrome. Sensitivity and specificity of tests for ocular and oral involvement in Sjögren's syndrome. Ann Rheum Dis 1994 53:637-47.

9 Bombardier C, Gladman DD, Urowitz MB, Caron D, Chang $\mathrm{CH}$, and the committee on prognosis studies in SLE. Derivation of SLEDAI. A disease activity index for lupus patients. Arthritis Rheum 1992;35:630-40.

10 Gladman DD, Urowitz MB, Fortin P, Isenberg DA, Goldsmith C, Gordon C, et al. Systemic Lupus International Collaborating Clinics conference on assessment of lupus flare and quality of life measures in SLE. Systemic lupus flare and quality of life measures in SLE. Systemic Lupus International Co

11 Gilboe IM, Kvien TK, Husby G. Health status in systemic lupus erythematosus compared to rheumatoid arthritis and healthy controls. J Rheumatol 1999;26:1694-700.

12 Smolen J, Strand V, Cardiel M, Edworthy SM, Furst D, Gladman D, et al. Randomized clinical trials and longitudinal observational studies in systemic lupus erythematosus: consensus on a preliminary core set of outcome domains. J Rheumatol 1999;26:504-7.

13 Sutcliffe N, Stoll T, Pyke S, Isenberg DA. Functional disability and end organ damage in patients with systemic (SS), and primary SS. J Rheumatol 1998;25:63-8.

14 Sjögren H, Bloch K. Keratoconjuntivitis sicca and the Sjögren's syndrome. Surv Ophthalmol 1971;16:145-59.

15 Kvien TK, Glennås A, Knudsrød OG, Smedstad LM, Mowinkel P, Førre Ø. The prevalence and severity of rheumatoid arthritis in Oslo: result from a county register and a matoid arthritis in Oslo: result from a county register and

population survey. Scand J Rheumatol 1997;26:412-18.
16 Uhlig T, Kvien TK, Glennås A, Smedstad LM, Førre Ø The incidence and severity of rheumatoid arthritis. Results from a county register in Oslo, Norway. J Rheumatol 1998; 25:1078-84

17 Tan EM, Cohen AS, Fries JF, Alfonse TM, McShane DJ, Rothefield NF, et al. The 1982 revised criteria for the classification of systemic lupus erythematosus. Arthritis Rheum 1982;25:1271-77

18 Gilboe IM, Husby G. Application of the 1982 revised ARA criteria for the classification of systemic lupus erythematosus on a cohort of 346 Norwegian patients with connective tissue disease. Scand J Rheumatol 1998;27:81-7.

19 Arnett FC, Edworthy SM, Bloch AB, McShane DJ, Fries JF, Cooper NS. The American Rheumatism Association 1987 revised criteria for the classification of rheumatoid arthritis. Arthritis Rheum 1988;31:315-24.

20 Gladman D, Ginzler E, Goldsmith C, Fortin P, Liang M, Urowitz $\mathrm{M}$, et al. The development and initial validation of the Systemic Lupus International Collaborating Clinics the Systemic Lupus International Collaborating Clinics American College of Rheumatology damage index for systemic 1 363-9.

21 Uhlig T, Kvien TK, Liaaen Jensen J, Axell T. Sicca symptoms, saliva and tear production, and disease variables in 636 patients with rheumatoid arthritis. Ann Rheum Dis 1999;58:415-22.

22 Pincus T, Callahan LF, Brooks RH, Fuchs HA, Olsen NJ, Kaye JJ. Assessment of patient satisfaction in activities of daily living using a modified Stanford Health Assessment Questionnaire. Arthritis Rheum 1983;26:1346-53.

23 Ware JE, Sherbourne CD. The MOS 36-item Short Form Health Survey (SF-36) I. Conceptual framework and item selection. Med Care 1992;30:473-83.

24 Workshop on diagnostic criteria for Sjögren's syndrome I Questionnaires for Sjögren's syndrome. II. Manual of methods and procedures. Clin Exp Rheumatol 1989;7: methods

25 Jensen J, Bergem H, Gilboe IM, Husby G, Axell T. Oral and ocular sicca symptoms and findings are prevalent in
systemic lupus erythematosus. J Oral Pathol Med 1999;28: systemic $317-22$.

26 Sreebny L, Valdini A, Brook S. Xerostomia. Part 1: Relationship to other oral symptoms and salivary gland hypofunction. Oral Surg Oral Med Oral Pathol 1988;66: $451-8$.

27 Friedlaender M. Ocular manifestations of Sjögren's sicca. Rheum Dis Clin North Am 1992;18:591-608.

28 Sreebny L, Valdini A, Yu A, Brook S. Xerostomia. Part II: Relationship to nonoral symptoms, drugs, and diseases. Oral Surg Oral Med Oral Pathol 1989;68:419-27.

29 Sreebny L, Schwartz S. A reference guide to drugs and dry mouth. Gerodontology 1986:5:75-99.

30 Alarcon-Segovia D, Ibáñez G, Velázques-Forero F, Hernández-Ortíz J, González-Jiménez Y. Sjögren's synHernandez-Ortiz J, Gonzalez-Jimenez Y. Sjögren's syn-
drome in systemic lupus erythematosus. Clinical and drome in systemic lupus erythematosus. Clinical and
subclinical manifestations. Ann Intern Med 1974;81:57783 .
31 Jonsson H, Nived O, Sturfelt G, Norberg R. Symptomatic secondary Sjögren's syndrome in patients with systemic lupus erythematosus. Scand J Rheumatol Suppl 1986;61: $166-9$.

32 Moutsopoulos HM, Klippel J, Pavlidis N, Wolf R, Sweet J, Steinberg A, et al. Correlative histologic and serologic findings of sicca syndrome in patients with systemic lupus erythematosus. Arthritis Rheum 1980;23:36-40.

33 Grennan D, Ferguson M, Williamson J, Dick W, Buchanan W. Sjögren's syndrome in SLE: Part 2. An examination of the clinical significance of Sjögren's syndrome by comparison of its frequency in typical and atypical forms of SLE, overlap syndrome and scleroderma. N Z Med J 1977;86: 376-9.

34 Seal D. The effect of ageing and disease on tear constituents. Trans Ophthalmol Soc UK 1985;104:355-62.

35 Gutman D, Ben-Aryeh H. The influence of age on salivary content and rate of flow. Int J Oral Surg 1974;3:314-17.

36 Pedersen W, Schubert M, Izutsu K, Mersai T, Truelove E. Age-dependent decrease in human submandibular gland
flow rates as measured under resting and post-stimulation conditions. J Dent Res 1985;64:822-5.

37 Brown C. The parotid puzzle: a review of the literature on human salivation and its applications to psychophysiology. Psychophysiology 1970;7:66-85.

38 Conner S, Iranpour B, Mills J. Alteration in parotid salivary flow in diabetes mellitus. Oral Surg 1970;30:55-9.

39 Andonopoulos A, Drosos A, Skopouli FN, Acritidis N, Moutsopoulos HM. Secondary Sjögren's syndrome in rheumatoid arthritis. J Rheumatol 1987;14:1098-103.

40 Andonopoulos A, Drosos A, Skopouli F, Moutsopoulos H. Sjögren's syndrome in rheumatoid arthritis and progressive systemic sclerosis. A comparative study. Clin Exp Rheumatol 1989;7:203-5.

41 Talal N. Sjögren's syndrome: historical overview and clinical spectrum of disease. Rheum Dis Clin North Am 1992;18: 507-15.

42 Dawes C. Physiological factors affecting salivary flow rate, oral sugar clearance, and the sensation of dry mouth in man. J Dent Res 1987;66:648-53.

43 Hay EM, Thomas E, Pal B, Hajeer A, Chambers H, Silman A. Weak association between subjective symptoms of and objective testing for dry eyes and mouth: result from population based study. Ann Rheum Dis 1998;57:20-4.

44 Jonsson H, Nived O, Sturfelt G. Outcome in systemic lupus erythematosus: a prospective study of patients from a defined population. Medicine (Baltimore) 1989;68:14150 .

45 Osial T, Whiteside T, Buckingham R, Singh G, Barnes L, Pierce J, et al. Clinical and serologic study of Sjögren's syndrome in patients with progressive systemic sclerosis. Arthritis Rheum 1983;26:500-8.

46 Jacobsson L, Hansen B, Manthorpe R, Handgrave K, Neas B, Harley J. Association of dry eyes and dry mouth with anti-Ro/SS-A and anti-La/SS-B autobodies in normal adults. Arthritis Rheum 1992;35:1492-501.

47 Krupp LB, LaRossa NG, Muir J, Steinberg AD. A study of fatigue in systemic lupus erythematosus. J Rheumatol 1990;17:1450-2.

48 Utset Olsen T, Golden M, Siberry G, Kiri N, Crum R, Petri M. Depressive symptoms in patients with systemic lupus erythematosus: association with central nervous system lupus and Sjögren's syndrome. J Rheumatol 1994;21: 2039-45.

49 Rhodus N, Johnson D. The prevalence of oral manifestations of systemic lupus erythematosus. Oral Pathol tions of syste

50 Schiødt M. Oral manifestations of lupus erythematosus. Int J Oral Surg 1984;13:101-47.

51 Jonsson R. Oral manifestation of systemic lupus erythematosus. Göteborg, Sweden: University of Göteborg, 1983.

52 Bell D. SLE in the elderly - Is it really SLE or systemic Sjögren's syndrome? J Rheumatol 1988;15:723-4.

53 van Bijsterveld $\mathrm{O}$. Diagnostic tests in the sicca syndrome. Arch Ophthalmol 1969;82:10-4.

54 van Bijsterveld O. Diagnosis and differential diagnosis of keratoconjunctivitis sicca associated with tear gland degeneration. Clin Exp Rheumatol 1990;8(suppl 5):3-6.

55 Paschides C, Kitsios G, Karakostas K, Psillas C, Moutsopoulos H. Evaluation of tear break-up time, Schirmer's-I test and rose bengal staining as confirmatory for keratoconjunctivitis sicca. Clin Exp Rheumatol 1989;7:155-7.

56 Jacobsson L, Axell T, Hansen B, Henricsson V, Larsson Å, Lieberkind K, et al. Dry eyes or mouth - an epidemiological study in Swedish adults, with special reference to primary Sjögren's syndrome. J Autoimmun 1989;2:521-7.

57 Dafni U, Tzioufas A, Staikos P, Skopouli F, Moutsopoulos $\mathrm{H}$. Prevalence of Sjögren's syndrome in a closed rural community. Ann Rheum Dis 1997;56:521-5.

58 Ben-Aryeh H, Gordon N, Szargel R, Toubi E, Laufer D. Whole saliva in systemic lupus erythematosus patients. Oral Surg Med Oral Pathol 1993;75:696-9.

59 Jonsson R, Bratthall D, Nyberg G. Histologic and sialochemical findings indicating sicca syndrome in patients with systemic lupus erythematosus. Oral Surg Oral Med Oral Pathol 1982;54:635-9. 